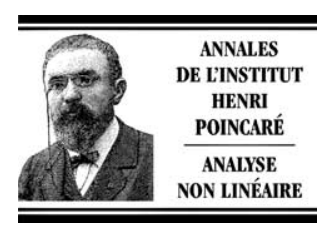

www.elsevier.com/locate/anihpc

\title{
Droplet spreading under weak slippage: the waiting time phenomenon
}

\author{
Günther Grün \\ Institut für Angewandte Mathematik, Universität Bonn, Beringstr. 4-6, 53115 Bonn, Germany \\ Received 6 March 2002; accepted 7 February 2003
}

\begin{abstract}
We prove the occurrence of a waiting time phenomenon in space dimensions $N<4$ for the thin film equation subjected to Navier's slip condition or even weaker slip conditions. We state a sufficient criterion on the smoothness of initial data which guarantees a local delay of the onset of spreading wherever the support of initial data locally satisfies an exterior cone condition. Our method combines a Hardy-type inequality valid on infinite cones with recently established weighted energy estimates and a novel iteration technique developed in [R. Dal Passo, L. Giacomelli, G. Grün, Ann. Scuola Norm. Sup. Pisa 30 (2001) 437-463]. On account of formal considerations, we conjecture our criterion to be optimal.

(C) 2004 L'Association Publications de l'Institut Henri Poincaré. Published by Elsevier B.V. All rights reserved
\end{abstract}

\section{Résumé}

Dans cet article nous nous intéressons au comportement qualitatif de la frontière libre des solutions de l'équation des films minces soumise aux conditions de glissement de type Navier ou aux conditions de glissement plus faibles. Nous montrons l'apparition d'un phénomène de temps d'attente dans les dimensions d'espace $N<4$. Nous formulons un critère suffisant pour la régularité des données initiales, qui garantit un délai local pour le début de la propagation, où que soit satisfaite localement une condition cônique extérieure par le support des données initiales. Notre méthode combine une inégalité de type Hardy valide sur un cône infini avec des estimations récentes pondérées d'énergie, et avec une nouvelle technique d'itération développée dans [R. Dal Passo, L. Giacomelli, G. Grün, Ann. Scuola Norm. Sup. Pisa 30 (2001) 437-463]. En raison de considérations formelles, nous formons la conjecture que notre critère est optimal.

(C) 2004 L'Association Publications de l'Institut Henri Poincaré. Published by Elsevier B.V. All rights reserved

Keywords: Fourth order degenerate parabolic equations; Waiting time phenomenon; Thin films

\section{Introduction}

In this paper, we derive a criterion on the smoothness of initial data which guarantees the occurrence of a waiting time phenomenon for strong solutions to the Cauchy problem associated with the fourth order degenerate parabolic equation

$$
u_{t}+\operatorname{div}\left(|u|^{n} \nabla \Delta u\right)=0 \quad \text { in } \mathbb{R}^{N} \times \mathbb{R}^{+}
$$

\footnotetext{
E-mail address: gg@iam.uni-bonn.de (G. Grün).
} 
in space dimensions $N<4$ for parameters $n \in[2,3)$. Equipped with non-negative, integrable initial data having $H^{1}$-regularity, Eq. (1.1) is a model problem for a class of fourth order degenerate parabolic equations that arise in materials sciences and fluid dynamics (cf. [5,8,19] and the references therein). In the version given above, $u$ describes the thickness of a thin film of viscous liquid that spreads on a horizontal surface under the influence of surface tension. In general $n$ is chosen to be a positive number. The particular values $n=2$ and $n=3$ are distinguished from a physical point of view. With a grain of salt, the exponent $n=2$ corresponds to the assumption of Navier's slip condition at the liquid-solid-interface. ${ }^{1}$ In the physical literature, also weaker slip conditions are suggested (cf. [2] and the references therein) which entail exponents $2<n<3$. The parameter $n=3$, however, is associated with a no slip condition and plays the role of a borderline value in the theory of Eq. (1.1). For $n \geqslant 3$, formal calculations (and rigorous results established for $n>4$ in one space dimension by Beretta, Bertsch and Dal Passo [1]) suggest that the solution's support is constant in time. For $0<n<3$, in contrast, solutions exist which spread and which have the property of finite speed of propagation. In a series of publications (see Bernis [3], Hulshof and Shishkov [16], Bertsch, Dal Passo, Garcke, Grün [4] and Grün [13]), it was possible to establish in all space dimensions $N<4$ and for $n \in(0,3)$ optimal estimates on the spreading rate.

Even more refined results on the behaviour of the free boundary, i.e. the boundary of $\operatorname{supp}(u(\cdot, t))$, can be obtained provided appropriate smoothness conditions on initial data are imposed. Dal Passo, Giacomelli and the author formulated in [6] in one space dimension (and for $n \in(0,2)$ also in space dimensions $N<4$ ) a sufficient criterion to guarantee the occurrence of a waiting time phenomenon. This means that for sufficiently small times the support locally does not increase. Surprisingly, both result and technique to be applied differ depending on whether $n \in(0,2)$ or $n \in[2,3)$. To put it briefly, in the former range a waiting time phenomenon occurs at a point $x_{0} \in \partial\left[\operatorname{supp}\left(u_{0}\right)\right]$ provided $u_{0}(x)$ grows at most like $\left|x-x_{0}\right|^{4 / n}$ in a neighborhood of $x_{0}$. In contrast, for $n \in[2,3)$ a slightly stronger condition has to be imposed which requires the derivative of initial data to satisfy locally

$$
\left|u_{0}^{\prime}(x)-u_{0}^{\prime}\left(x_{0}\right)\right| \leqslant C\left|x-x_{0}\right|^{4 / n-1}
$$

On a merely technical level, this may be explained by the fact that for $n \in(0,2)$ the reasoning is based on the so called $\alpha$-entropy estimate

$$
\frac{1}{\alpha(\alpha+1)} \int_{\mathbb{R}^{N}} u^{\alpha+1}(\cdot, T)+C^{-1} \int_{0}^{T} \int_{\mathbb{R}^{N}}\left\{\left|\nabla u^{(\alpha+n+1) / 4}\right|^{4}+\left|D^{2} u^{(\alpha+n+1) / 2}\right|^{2}\right\} \leqslant \frac{1}{\alpha(\alpha+1)} \int_{\mathbb{R}^{N}} u_{0}^{\alpha+1} .
$$

In the case of non-negative, not strictly positive initial data this estimate does only hold for

$$
\alpha \in\left(\max \left\{-1, \frac{1}{2}-n\right\}, 2-n\right) \backslash\{0\} .
$$

Note that condition (1.3) does not permit to choose the parameter $\alpha$ positive for values of $n \in[2,3)$. Therefore, it is not possible to control the entropy $u^{\alpha+1}$ at times $T>0$ in terms of the initial entropy, and the entropy estimate seems to be inappropriate to yield results on the qualitative behaviour of the free boundary. Hence, in the parameter regime $n \in[2,3)$ the argumentation has to be based upon other tools. As comparison principles do not hold for fourth order parabolic equations, the remedy seems to be to exploit the only remaining integral estimate which is the energy estimate

$$
\frac{1}{2} \int_{\Omega}|\nabla u(\cdot, T)|^{2}+\int_{\Omega_{T}} u^{n}|\nabla \Delta u|^{2} \leqslant \frac{1}{2} \int_{\Omega}\left|\nabla u_{0}\right|^{2} .
$$

\footnotetext{
${ }^{1}$ More precisely, Navier's slip condition entails a nonlinearity $m(u):=u^{3}+\beta u^{2}$, where the positive parameter $\beta$ is the slip length (cf. [19]). However, the qualitative behavior of solutions is governed by the smoothness of $m(\cdot)$ in its point of degeneracy. Therefore, we may confine ourselves to the case $m(u)=u^{2}$.
} 
Apparently, it provides estimates only in terms of the gradient of initial data.

In contrast to the parameter regime $n \in(0,2)$ where the one-dimensional techniques were generalized to the multi-dimensional case with at most small a time delay, the situation turned out to be much more intricate for $n \in[2,3)$. Indeed, questions about finite speed of propagation or occurrence of a waiting time phenomenon in higher space dimensions remained open for quite a while. This was particularly unsatisfactory since the case $n=2$ corresponds to the assumption of Navier's slip condition which is - according to Jäger and Mikelic [17] - the effective boundary condition for laminar flow over rough surfaces. The main obstacle was to make the term $\int u^{n}|\nabla \Delta u|^{2}$, which physically represents the dissipated energy, accessible to Gagliardo-Nirenberg-type interpolation arguments. This hurdle was overcome by virtue of the interpolation inequality

$$
\int_{\Omega}\left|\nabla u^{(n+2) / 6}\right|^{6}+\int_{\Omega}\left|\nabla \Delta u^{(n+2) / 2}\right|^{2} \leqslant C(n, N) \int_{\Omega} u^{n}|\nabla \Delta u|^{2}
$$

which holds on convex domains $\Omega$ for positive functions of class $H^{2}$ having zero normal derivative on the boundary and which was proven in Grün [12] (see also the recently published paper Grün [11]). This estimate was the key ingredient to establish in [12] the existence of strong solutions to the Cauchy problem in multiple space dimensions which satisfy besides the $\alpha$-entropy estimate in particular a weighted version of the energy estimate (1.4). Moreover, it was the starting point to prove qualitative and quantitative results on finite speed of propagation for the solution to the Cauchy problem constructed in [12]. And implicitly, it will also serve as a main tool to formulate a sufficient criterion for the occurrence of a waiting time phenomenon in this paper.

A simplified version of our result reads as follows. Assume the existence of an infinite cone $\mathcal{C}\left(x_{0}, 2 \theta\right)$ with vertex $x_{0} \in \partial\left[\operatorname{supp}\left(u_{0}\right)\right]$ and with opening angle $2 \theta$ such that

$$
\mathcal{C}\left(x_{0}, 2 \theta\right) \cap \operatorname{supp}\left(u_{0}\right)=\emptyset .
$$

There is a finite time $T_{*}>0$ such that the solution stays zero on a cone $\mathcal{C}\left(x_{0}, \theta\right)$ having the same symmetry axis as $\mathcal{C}\left(x_{0}, 2 \theta\right)$ provided $\nabla u_{0}$ satisfies an estimate of the form

$$
\left|\nabla u_{0}(x)-\nabla u_{0}\left(x_{0}\right)\right| \leqslant C\left|x-x_{0}\right|^{4 / n-1}
$$

within a neighborhood of $x_{0}$.

On account of formal considerations, partially already presented in [6], we believe the exponent $\gamma=4 / n$ to be optimal. However, it remains open whether the condition on the derivative can be replaced by the slightly weaker condition

$$
\left|u_{0}(x)-u_{0}\left(x_{0}\right)\right| \leqslant C\left|x-x_{0}\right|^{4 / n}
$$

which involves values of the function $u_{0}$ only.

Let us describe our method and the outline of the paper. The result is based on a new Hardy-type inequality valid on infinite cones, it is based on the recently established, aforementioned weighted energy estimate and on the novel technique developed together with Dal Passo and Giacomelli in [6]. In Section 2, we will summarize the properties of the strong solutions to the Cauchy problem established in [12]. Section 3 is devoted to the proof of the announced new Hardy-type inequality valid on cones. Combining that inequality with the weighted energy estimate of Section 2, the first ingredient for our method is readily prepared. In Section 4 we state and prove the main result of this paper. Hereby, we take advantage of a number of auxiliary results, for instance an iteration lemma formulated in [6] reminiscent of Stampacchia's lemma. All these auxiliary tools will be listed in the appendix. Finally, we sketch a refined qualitative result on finite speed of propagation in Section 5 which uses the new Hardy-type inequality for cones and which hence permits to provide local results also in the case of initial data having non-convex support.

Throughout the paper, we will use the standard notation for Sobolev spaces. With a slight misuse of notation, we write $\|u\|_{p}$ for $\left(\int|u|^{p}\right)^{1 / p}$ also in the case $0<p<1 .\left\{e_{1}, \ldots, e_{N}\right\}$ denotes the canonical basis of $\mathbb{R}^{N}, x_{i}$, $i=1, \ldots, N$, are the coordinates of an element $x \in \mathbb{R}^{N}$ with respect to that basis. Sometimes, we will decompose $x=x_{N} \cdot e_{N}+\bar{x}_{N}$. Both $B(x, R)$ and $B_{R}(x)$ denote the ball with radius $R$ around $x$. Finally, we will write $[u>0]_{T}$ for $\left\{(x, t) \in \mathbb{R}^{N} \times(0, T) \mid u(x, t)>0\right\}$. 


\section{Definition and properties of strong solutions}

Eq. (1.1) together with non-negative, compactly supported initial data of class $H^{1}$ implicitly defines a free boundary problem where the free boundary is given by $\partial[\operatorname{supp}(u(\cdot, t))]$. Since the governing equation is fourth order, we cannot expect solutions to be unique unless three conditions are prescribed at the free boundary. Therefore, the natural conditions

$$
\begin{aligned}
& \left.u\right|_{\partial[\operatorname{supp}(u(\cdot, t))]}=0, \\
& \left.u^{n} \frac{\partial}{\partial \nu} \Delta u\right|_{\partial[\operatorname{supp}(u(\cdot, t))]}=0 \quad \text { (conservation of mass) }
\end{aligned}
$$

( $v$ the outer normal to $\operatorname{supp}(u(\cdot, t)))$ have to be supplemented by a third one. A possible choice might be a condition on the normal derivative - or to put it in physical terms - a condition on the contact angle. With the exception of the work of Otto [20] on fixed-nonzero-contact-angle-solutions in one space dimension for the Hele-Shaw problem $(n=1)$, the analytic work concentrated so far on so called strong solutions, i.e. solutions for which a zero-contact angle has implicitly been imposed via the entropy estimate (1.2). In [12], we proved the following existence result for strong solutions to the Cauchy problem in the multi-dimensional case.

Theorem 2.1. Let $n \in(2-\sqrt{1-N /(8+N)}, 3), N<4$, and assume $u_{0} \in H^{1}\left(\mathbb{R}^{N}\right)$ to be non-negative with compact support in the sense that $u_{0}(x)=0$ almost everywhere on $\mathbb{R}^{N} \backslash B_{R_{0}}(0)$ for a positive number $R_{0}$. Then, a non-negative function $u$ exists that has the following properties:

(i) Regularity:

$$
\begin{aligned}
& u_{t} \in L^{2}\left(\mathbb{R}^{+} ;\left(W^{1, p}(\Omega)\right)^{\prime}\right) \quad \text { for } p>\frac{4 N}{2 N+n(2-N)} \text { and any } \Omega \Subset \mathbb{R}^{N}, \\
& u \in L^{\infty}\left(\mathbb{R}^{+} ; H^{1}\left(\mathbb{R}^{N}\right)\right), \\
& \nabla \Delta u^{(n+2) / 2} \in L^{2}\left(\mathbb{R}^{N} \times \mathbb{R}^{+}\right), \\
& \nabla u^{(n+2) / 6} \in L^{6}\left(\mathbb{R}^{N} \times \mathbb{R}^{+}\right), \\
& D^{2} u^{(\alpha+n+1) / 2} \in L^{2}\left(\mathbb{R}^{N} \times \mathbb{R}^{+}\right) \quad \text { for any } \alpha \in(\max \{-1,1 / 2-n\}, 2-n), \\
& \nabla u^{(\alpha+n+1) / 4} \in L^{4}\left(\mathbb{R}^{N} \times \mathbb{R}^{+}\right) \quad \text { for any } \alpha \in(\max \{-1,1 / 2-n\}, 2-n), \\
& J=\left\{\begin{array}{cc}
u^{n} \nabla \Delta u & \text { on }[u>0]_{T} \\
0 \quad & \text { on }[u=0]_{T} \\
4 N
\end{array} \quad \in L^{2}\left(\mathbb{R}^{+} ; L^{q}\left(\mathbb{R}^{N}\right)\right)\right. \\
& \text { for any } 1<q<\frac{4 .}{2 N+n(N-2)} .
\end{aligned}
$$

(ii) $u$ is a solution to the Cauchy problem in the sense that

$$
\int_{0}^{T}\left\langle u_{t}, \phi\right\rangle_{\left(W^{1, p}(B(0))\right)^{\prime} \times W^{1, p}(B(0))}-\int_{[u>0]_{T}} u^{n} \nabla \Delta u \nabla \phi=0
$$

for $p>\frac{4 N}{2 N+n(2-N)}$, arbitrary $T>0$ and for all test functions $\phi$ contained in $L^{2}\left((0, T) ; W^{1, \infty}\left(\mathbb{R}^{N}\right)\right)$ such that $\bigcup_{t \in(0, T)} \operatorname{supp}(\phi(\cdot, t)) \subset B(0)$, where $B(0)$ is an arbitrary ball centered in the origin $0 \in \mathbb{R}^{N}$. In particular, initial data $u_{0}$ are attained continuously in time with respect to the $L^{\beta}$-norm for all $1 \leqslant \beta<\frac{2 N}{N-2}$.

Remark. (1) The condition on $n$ is a consequence of the validity range of inequality (1.5). 
(2) The reason for the restriction of the spatial dimension to values $N<4$ is rather subtle. It is connected with results on compactness in time of solutions to approximating auxiliary problems. For more details, we refer the reader to [12] (or to [5] where similar restrictions can be found).

In addition, the following quantitative result on finite speed of propagation holds which was established in [13].

Theorem 2.2. Let $u$ be a solution to the Cauchy problem associated with Eq. (1.1) as constructed in Theorem 2.1. Then a positive constant $C$ exists which only depends on $n, N$ and the mass of initial data such that

$$
\operatorname{supp} u(\cdot, t) \subset B\left(0, R_{0}+C \cdot t^{1 /(4+n N)}\right)
$$

for all $t>0$.

Remark. This result is optimal in the sense that the exponent $\sigma=\frac{1}{4+n N}$ is identical with the exponent that determines the radial growth rate of the support of self-similar solutions which were studied by Ferreira and Bernis [9].

For the purposes of this paper, we need the following corollary.

Corollary 2.3. Suppose in addition to the assumptions of Theorem 2.1 that $\psi$ is a non-negative function in $C^{2}\left(\mathbb{R}^{N}\right)$. Then, a positive constant $C_{2}=C_{2}(n, N)$ exists such that the following weighted energy estimate is satisfied by the solution u constructed in Theorem 2.1 .

$$
\begin{array}{r}
\int_{\mathbb{R}^{N}} \psi^{6}|\nabla u(\cdot, T)|^{2}+C_{2}^{-1}\left\{\int_{0}^{T} \int_{\mathbb{R}^{N}} \psi^{6}\left|\nabla u^{(n+2) / 6}\right|^{6}+\int_{0}^{T} \int_{\mathbb{R}^{N}} \psi^{6}\left|\nabla \Delta u^{(n+2) / 2}\right|^{2}\right\} \\
\leqslant \int_{\mathbb{R}^{N}} \psi^{6}\left|\nabla u_{0}\right|^{2}+C_{2} \int_{0}^{T} \int_{\mathbb{R}^{N}} u^{n+2}\left\{|\nabla \psi|^{6}+\left|D^{2} \psi\right|^{2}|\nabla \psi|^{2} \psi^{2}+\left|D^{2} \psi\right|^{3} \psi^{3}\right\}
\end{array}
$$

for arbitrary $T>0$.

Proof. In [12], Theorem 6.1.1, a similar result was established on bounded convex domains $\Omega$ provided the tangential component of $\nabla \psi$ vanishes on $\partial \Omega$. In the case of interest here -i.e. $\Omega=\mathbb{R}^{N}$ and no requirements to be imposed on the tangential component of $\nabla \psi$ on the boundary of bounded domains - we may use an approximation argument based on the finite speed of propagation property of $u$ as established in Theorem 2.2. For given $T>0$, we choose the radius $\bar{R}(T)>0$ such large that

$$
\bigcup_{t \in(0, T)} \operatorname{supp}(u(\cdot, t)) \subset B(\bar{R}(T)) .
$$

Taking a smooth non-negative localization function $\varphi_{T}$ with the properties

$$
\begin{array}{ll}
\varphi_{T} \equiv 1 & \text { on } B(\bar{R}(T)), \\
\varphi_{T} \equiv 0 & \text { on } B(2 \bar{R}(T))
\end{array}
$$

and choosing the test-function $\psi(x) \cdot \varphi_{T}(x)$ - which is admissible - a predecessor of (2.19) with right-hand side given by

$$
\int_{0}^{T} \int_{\mathbb{R}^{N}} u^{n+2}\left\{\left|\nabla\left(\psi \varphi_{T}\right)\right|^{6}+\left|D^{2}\left(\psi \varphi_{T}\right)\right|^{2}\left|\nabla\left(\psi \varphi_{T}\right)\right|^{2}\left|\psi \varphi_{T}\right|^{2}+\left|D^{2}\left(\psi \varphi_{T}\right)\right|^{3}\left|\psi \varphi_{T}\right|^{3}\right\}
$$


can easily be established along the lines of proof of Theorem 6.2.1 in [12]. By virtue of the finite-speed-ofpropagation of $u$, the assertion follows.

\section{A Hardy-type inequality valid on infinite cones}

In this section, we present a new Hardy-type inequality valid on infinite cones which will be an essential tool for the results to follow. Before stating and proving it, let us recall the classical Hardy inequality (for a proof see $[14,15]$ and the monograph [18]):

Lemma 3.1. Let $-\infty \leqslant a<b \leqslant \infty$ be real numbers and assume that $1 \leqslant p \leqslant q \leqslant \infty$. For weight functions $v, w$ which are non-negative and measurable on $(a, b)$, consider the quantities

$$
F_{\widetilde{R}}(x):=F_{\widetilde{R}}(x ; a, b, w, v, q, p)=\left\|w^{1 / q}\right\|_{q,(a, x)} \cdot\left\|v^{-1 / p}\right\|_{p^{\prime},(x, b)}
$$

and

$$
A_{\widetilde{R}}:=A_{\widetilde{R}}(a, b, w, v, q, p)=\sup _{a<x<b} F_{\widetilde{R}}(x) .
$$

Then for every

$$
u \in A C_{\widetilde{R}}(a, b):=\left\{u \in W_{\mathrm{loc}}^{1,1}(a, b): \lim _{x \supset b} u(x)=0\right\},
$$

the Hardy-inequality

$$
\left\|u \cdot w^{1 / q}\right\|_{q,(a, b)} \leqslant C_{\widetilde{R}}\left\|u_{x} \cdot v^{1 / p}\right\|_{p,(a, b)}
$$

holds, if and only if $A_{\widetilde{R}}<\infty$.

Moreover, the best possible constant $C_{\widetilde{R}}$ in (3.1) satisfies the estimate

$$
A_{\widetilde{R}} \leqslant C_{\widetilde{R}} \leqslant k(q, p) \cdot A_{\widetilde{R}}
$$

where

$$
k(q, p):=\left(1+\frac{q}{p^{\prime}}\right)^{1 / q}\left(1+\frac{p^{\prime}}{q}\right)^{1 / p^{\prime}} .
$$

Our result reads as follows. To avoid unnecessary technicalities, we formulate it for a cone with symmetry axis given by the $x_{N}$-axis.

Lemma 3.2. Consider the cone $\mathcal{C}(y, \theta):=\left\{x \in \mathbb{R}^{N}:\left|\bar{x}_{N}-\bar{y}_{N}\right|<\left(x_{N}-y_{N}\right) \tan \theta\right\}$ with opening angle $\theta \in(0, \pi / 2)$ and vertex $y \in \mathbb{R}^{N}$. Let $\bar{\zeta}_{y}: \mathcal{C}(y, \theta) \rightarrow \mathbb{R}_{0}^{+}$be defined as

$$
\bar{\zeta}_{y}\left(\bar{x}_{N}, x_{N}\right):=\left(x_{N}-y_{N}\right)\left(\tan ^{2} \theta-\frac{\left|\bar{x}_{N}-\bar{y}_{N}\right|^{2}}{\left(x_{N}-y_{N}\right)^{2}}\right) .
$$

Then a constant $K=K(\theta)$ exists such that

$$
\int_{\mathcal{C}(y, \theta)} \bar{\zeta}_{y}^{4} \cdot u^{2} \leqslant K(\theta) \cdot \int_{\mathcal{C}(y, \theta)} \bar{\zeta}_{y}^{6}\left|\frac{\partial u}{\partial x_{N}}\right|^{2}
$$

for arbitrary $u \in H^{1}(\mathcal{C}(y, \theta))$. 
Proof. W.l.o.g. we may assume $y=0$, and we abbreviate $\beta:=\tan \theta$. Let us apply the one-dimensional Hardyinequality (3.1) along half-lines parallel to the $x_{N}$-axis. Abbreviating $\mathcal{C}(\theta):=\mathcal{C}(0, \theta)$, we have by virtue of Fubini's theorem that

$$
\int_{\mathcal{C}(\theta)} v(x) \mathrm{d} x=\int_{\mathbb{R}^{N-1}}\left(\int_{\left|\bar{x}_{N}\right| \cdot \beta^{-1}}^{\infty} v\left(\bar{x}_{N}, x_{N}\right) \mathrm{d} x_{N}\right) \mathrm{d} \bar{x}_{N} .
$$

Let us consider the quantities

$$
F_{\widetilde{R}}\left(z ; \bar{x}_{N}\right):=\left(\int_{\left|\bar{x}_{N}\right| \cdot \beta^{-1}}^{z} \bar{\zeta}^{4}\left(\bar{x}_{N}, x_{N}\right) \mathrm{d} x_{N}\right)^{1 / 2} \cdot\left(\int_{z}^{\infty} \bar{\zeta}^{-6}\left(\bar{x}_{N}, x_{N}\right) \mathrm{d} x_{N}\right)^{1 / 2}
$$

and

$$
A_{\widetilde{R}}\left(\bar{x}_{N}\right):=\sup _{y \in\left(\left|\bar{x}_{N}\right| / \beta, \infty\right)} F_{\widetilde{R}}\left(y ; \bar{x}_{N}\right) .
$$

In (3.7), we shortly wrote $\bar{\zeta}\left(\bar{x}_{N}, x_{N}\right)$ for $\bar{\zeta}_{0}\left(\bar{x}_{N}, x_{N}\right)$.

Assuming for the moment that

$$
K(\theta):=\operatorname{esssup}_{\bar{x}_{N} \in \mathbb{R}^{N-1}} A_{\widetilde{R}}\left(\bar{x}_{N}\right)<\infty,
$$

we conclude by virtue of (3.1) as follows:

$$
\begin{aligned}
\int_{\mathcal{C}(\theta)} \bar{\zeta}^{4} u^{2} & =\int_{\mathbb{R}^{N-1}}\left(\int_{\left|\bar{x}_{N}\right| \cdot \beta^{-1}} \bar{\zeta}^{4}\left(\bar{x}_{N}, x_{N}\right) \cdot u^{2}\left(\bar{x}_{N}, x_{N}\right) \mathrm{d} x_{N}\right) \mathrm{d} \bar{x}_{N} \\
& \leqslant K(\theta) \int_{\mathbb{R}^{N-1}}\left(\int_{\left|\bar{x}_{N}\right| \cdot \beta^{-1}} \bar{\zeta}^{6}\left(\bar{x}_{N}, x_{N}\right) \cdot\left|\frac{\partial u}{\partial x_{N}}\left(\bar{x}_{N}, x_{N}\right)\right|^{2} \mathrm{~d} x_{N}\right) \mathrm{d} \bar{x}_{N} \\
& =K(\theta) \int_{\mathcal{C}(\theta)} \bar{\zeta}^{6} \cdot\left|\frac{\partial u}{\partial x_{N}}\right|^{2} .
\end{aligned}
$$

It remains to prove (3.9). Writing

$$
\bar{\zeta}\left(\bar{x}_{N}, x_{N}\right)=\frac{x_{N}^{2} \beta^{2}-\left|\bar{x}_{N}\right|^{2}}{x_{N}}=\beta^{2}\left(x_{N}-\left|\bar{x}_{N}\right| \cdot \beta^{-1}\right) \cdot \frac{x_{N}+\left|\bar{x}_{N}\right| \beta^{-1}}{x_{N}}
$$

and recalling that

$$
x_{N}>\frac{\left|\bar{x}_{N}\right|}{\beta}
$$

on $\mathcal{C}(\theta)$, we estimate easily

$$
\beta^{2}\left(x_{N}-\frac{\left|\bar{x}_{N}\right|}{\beta}\right) \leqslant \bar{\zeta}\left(\bar{x}_{N}, x_{N}\right) \leqslant 2 \beta^{2}\left(x_{N}-\frac{\left|\bar{x}_{N}\right|}{\beta}\right)
$$

on $\mathcal{C}(\theta)$.

Hence,

$$
F_{\widetilde{R}}\left(z ; \bar{x}_{N}\right)^{2} \leqslant 16 \cdot \beta^{8} \int_{\left|\bar{x}_{N}\right| \beta^{-1}}^{z}\left(x_{N}-\frac{\left|\bar{x}_{N}\right|}{\beta}\right)^{4} \mathrm{~d} x_{N} \cdot \beta^{-12} \int_{z}^{\infty}\left(x_{N}-\frac{\left|\bar{x}_{N}\right|}{\beta}\right)^{-6} \mathrm{~d} x_{N}=\frac{16 \beta^{-4}}{25} .
$$


This proves the assertion of the lemma.

For further purposes, we will need the following

Lemma 3.3. Consider for $r \in \mathbb{R}$ the function $\zeta_{r}: \mathbb{R}^{N} \rightarrow \mathbb{R}_{0}^{+}$defined by

$$
\zeta_{r}\left(\bar{x}_{N}, x_{N}\right):= \begin{cases}\left(x_{N}-r\right)\left[\tan ^{2} \theta-\frac{\left|\bar{x}_{N}\right|^{2}}{\left(x_{N}-r\right)^{2}}\right] & \text { on } \mathcal{C}\left(r \cdot e_{N}, \theta\right) \\ 0 & \text { on } \mathbb{R}^{N} \backslash \mathcal{C}\left(r \cdot e_{N}, \theta\right) .\end{cases}
$$

There exists a positive constant $C=C(\theta)$ such that

$$
\left|\nabla \zeta_{r}\right| \leqslant C
$$

and

$$
\left|D^{2} \zeta_{r}\right| \cdot \zeta_{r} \leqslant C
$$

on the whole of $\mathbb{R}^{N}$.

Proof. We use again the abbreviation $\beta:=\tan \theta$ and obtain

$$
\begin{aligned}
& \nabla \zeta_{r}(x)=\left(\begin{array}{c}
\frac{-2 \bar{x}_{N}}{x_{N}-r} \\
\beta^{2}+\frac{\left|\bar{x}_{N}\right|^{2}}{\left(x_{N}-r\right)^{2}}
\end{array}\right) \quad \text { on } \mathcal{C}\left(r \cdot e_{N}, \theta\right) \\
& D^{2} \zeta_{r}(x)=\left(\begin{array}{cc}
-\frac{2}{x_{N}-r} \mathbf{I d}_{N-1} & \frac{2 \bar{x}_{N}}{\left(x_{N}-r\right)^{2}} \\
\frac{2 \bar{x}_{N}}{\left(x_{N}-r\right)^{2}} & -\frac{2\left|\bar{x}_{N}\right|^{2}}{\left(x_{N}-r\right)^{3}}
\end{array}\right) \quad \text { on } \mathcal{C}\left(r \cdot e_{N}, \theta\right) .
\end{aligned}
$$

Hence,

$$
\left|\nabla \zeta_{r}(x)\right| \leqslant C\left(\frac{\left|\bar{x}_{N}\right|^{2}}{\left(x_{N}-r\right)^{2}}+\left(\beta^{2}+\frac{\left|\bar{x}_{N}\right|^{2}}{\left(x_{N}-r\right)^{2}}\right)^{2}\right)^{1 / 2} \text { on } \mathcal{C}\left(r \cdot e_{N}, \theta\right)
$$

and

$$
\left|\nabla \zeta_{r}\right|=0 \quad \text { on } \mathbb{R}^{N} \backslash \mathcal{C}\left(r \cdot e_{N}, \theta\right)
$$

On the other hand,

$$
\frac{\left|\bar{x}_{N}\right|^{2}}{\left(x_{N}-r\right)^{2}}<\beta^{2}
$$

for $x \in \mathcal{C}\left(r \cdot e_{N}, \theta\right)$ by construction and therefore

$$
\left|\nabla \zeta_{r}\right| \leqslant C(\beta)
$$

Estimate (3.13) follows in a similar fashion. 


\section{A sufficient criterion for the occurrence of a waiting time phenomenon}

In this section, we state and prove the main result of this paper. It reads as follows.

Theorem 4.1. Let $u$ be a solution of (1.1) to compactly supported, non-negative initial data having $H^{1}$-regularity as constructed in Theorem 2.1. Assume that $y$ is a boundary point of $\operatorname{supp}\left(u_{0}\right)$ and that the following properties hold:

- for a number $0<\theta<\pi / 4$, there is a cone $\mathcal{C}(y, 2 \theta):=\left\{x \in \mathbb{R}^{N}:\left|\bar{y}_{N}-\bar{x}_{N}\right|<\left(x_{N}-y_{N}\right) \cdot \tan 2 \theta\right\}$ with opening angle $2 \theta$ and vertex $y$ such that $\operatorname{supp}\left(u_{0}\right) \cap \mathcal{C}(y, 2 \theta)=\emptyset$,

- initial data satisfy

$$
\limsup _{r \rightarrow 0} r^{2-2 \gamma} f_{B(y, r)}\left|\nabla u_{0}\right|^{2} \mathrm{~d} x<\infty .
$$

Then the following is true: if $\gamma \geqslant 4 / n$, then the solution $u$ exhibits a waiting time phenomenon locally in $y$ in the following sense: there exists a positive time $T^{*}=T^{*}\left(n, N, u_{0}, \gamma, \theta\right)$ such that

$$
\operatorname{supp}(u(\cdot, t)) \cap \mathcal{C}(y, \theta)=\emptyset
$$

for $t \in\left[0, T^{*}\right)$.

Remark. (1) For notational simplicity, we only consider cones with symmetry axis given by the $x_{N}$-axis. The general case can easily be dealt with by an appropriate rotation argument.

(2) It is surprising that the result can be proven without using the $L^{2}$-bound on $\nabla \Delta u^{(n+2) / 2}$. Instead, we take advantage of the $L^{6}$-integrability of $\nabla u^{(n+2) / 6}$. This way, further technical difficulties related to interpolation arguments can be avoided. Moreover, note that formal calculations performed with $D^{3} u^{(n+2) / 2}$ yield the same result as presented in the theorem. This seems to be due to the fact that both bounds imply the same smoothness result for $u$ at the free boundary.

Proof. W.l.o.g. we assume $y=0$. Let us first fix a positive number $R_{1}$. Consider for $0<R<R_{1}$ the cones

$$
\mathcal{C}(-R):=\mathcal{C}\left(-\operatorname{Re}_{N}, \theta\right) \text {. }
$$

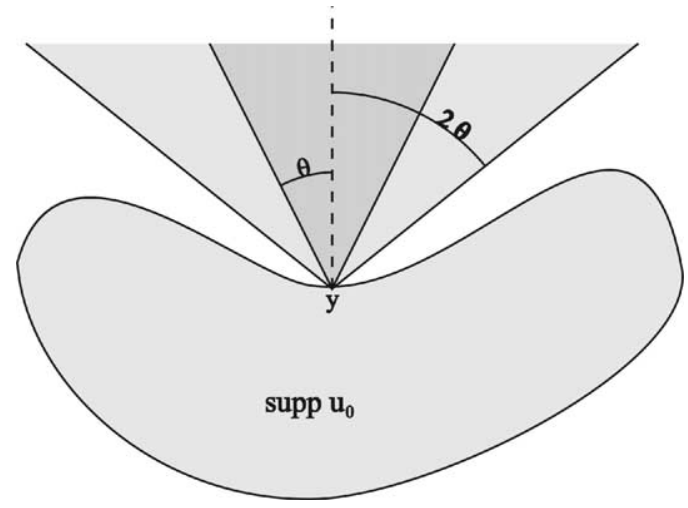

Fig. 1. Provided initial data are sufficiently smooth in a neighborhood of $y$, the solution $u$ stays zero for finite time within the inner cone. 
By virtue of elementary geometric reasoning, it becomes evident that

$$
\mathcal{C}(-R) \backslash \mathcal{C}(0,2 \theta) \subset B(0, R)
$$

for all $0<R<R_{1}$. Combining Corollary 2.3 with (3.12), (3.13) and with a straightforward approximation argument, the following key estimate can be obtained.

$$
\sup _{t \in(0, T)} \int_{\mathcal{C}(-R)} \zeta_{-R}^{6}|\nabla u(\cdot, t)|^{2}+C_{2}^{-1} \int_{0}^{T} \int_{\mathcal{C}(-R)} \zeta_{-R}^{6}\left|\nabla u^{(n+2) / 6}\right|^{6} \leqslant \int_{\mathcal{C}(-R)} \zeta_{-R}^{6}\left|\nabla u_{0}\right|^{2}+C_{2} \int_{0}^{T} \int_{\mathcal{C}(-R)} u^{n+2}
$$

for $0<R<R_{1}$ and arbitrary $T>0$.

By virtue of Hardy's inequality (3.5), we see that

$$
\int_{\mathcal{C}(-R)} \zeta_{-R}^{6}|\nabla u|^{2} \geqslant \int_{\mathcal{C}(-R)} \zeta_{-R}^{6}\left|\frac{\partial u}{\partial x_{N}}\right|^{2} \geqslant C(\theta) \int_{\mathcal{C}(-R)} \zeta_{-R}^{4} u^{2}
$$

hence,

$$
\sup _{t \in(0, T)} \int_{\mathcal{C}(-R)} \zeta_{-R}^{4} u^{2}+C_{2}^{-1} \int_{0}^{T} \int_{\mathcal{C}(-R)} \zeta_{-R}^{6}\left|\nabla u^{(n+2) / 6}\right|^{6} \leqslant \int_{\mathcal{C}(-R)} \zeta_{-R}^{6}\left|\nabla u_{0}\right|^{2}+C_{2} \int_{0}^{T} \int_{\mathcal{C}(-R)} u^{n+2} .
$$

On the other hand, we find for $0<\rho<R$ that

$$
\zeta_{-R}(x) \geqslant(R-\rho) \tan ^{2} \theta \text { for all } x \in \mathcal{C}(-\rho) .
$$

Hence,

$$
\begin{aligned}
& \sup _{t \in(0, T)} \int_{\mathcal{C}(-\rho)} u^{2}(\cdot, t)+(R-\rho)^{2} \int_{0}^{T} \int_{\mathcal{C}(-\rho)}\left|\nabla u^{(n+2) / 6}\right|^{6} \\
& \leqslant \frac{C}{(R-\rho)^{4}}\left\{\int_{\mathcal{C}(-R)} \zeta_{-R}^{6}\left|\nabla u_{0}\right|^{2}+\int_{0}^{T} \int_{\mathcal{C}(-R)} u^{n+2}\right\} .
\end{aligned}
$$

By virtue of an appropriate version of Gagliardo-Nirenberg's inequality (see Lemma A.3), we infer that

$$
\int_{0}^{T} \int_{\mathcal{C}(-R)} u^{n+2} \leqslant K_{1}\left(\int_{0}^{T} \int_{\mathcal{C}(-R)}\left|\nabla u^{(n+2) / 6}\right|^{6}\right)^{n N /(n N+12)}\left(\int_{0}^{T}\left(\int_{\mathcal{C}(-R)} u^{2}\right)^{(n+2) / 2}\right)^{12 /(n N+12)} .
$$

Young's inequality entails that

$$
\frac{1}{(R-\rho)^{4}} \int_{0}^{T} \int_{\mathcal{C}(-R)} u^{n+2} \leqslant \varepsilon(R-\rho)^{2} \int_{0}^{T} \int_{\mathcal{C}(-R)}\left|\nabla u^{(n+2) / 6}\right|^{6}+C_{\varepsilon}(R-\rho)^{-(4+n N / 2)} \int_{0}^{T}\left(\int_{\mathcal{C}(-R)} u^{2}\right)^{(n+2) / 2},
$$

altogether:

$$
\sup _{t \in(0, T)} \int_{\mathcal{C}(-\rho)} u^{2}(\cdot, t)+(R-\rho)^{2} \int_{0}^{T} \int_{\mathcal{C}(-\rho)}\left|\nabla u^{(n+2) / 6}\right|^{6}
$$




$$
\begin{aligned}
\leqslant & \varepsilon(R-\rho)^{2} \int_{0}^{T} \int_{\mathcal{C}(-R)}\left|\nabla u^{(n+2) / 6}\right|^{6}+C_{\varepsilon}(R-\rho)^{-(4+n N / 2)} \int_{0}^{T}\left(\int_{\mathcal{C}(-R)} u^{2}\right)^{(n+2) / 2} \\
& +\frac{C}{(R-\rho)^{4}} \int_{\mathcal{C}(-R)} \zeta_{-R}^{6}|\nabla u|^{2} .
\end{aligned}
$$

Introducing

$$
\begin{aligned}
& V(\rho):=\sup _{t \in(0, T)} \int_{\mathcal{C}(-\rho)} u^{2}(\cdot, t), \\
& U(\rho):=\int_{0}^{T} \int_{\mathcal{C}(-\rho)}\left|\nabla u^{(n+2) / 6}\right|^{6}, \\
& F_{\varepsilon}(\rho, R):=\frac{C_{\varepsilon}}{(R-\rho)^{4+n N / 2}} \int_{0}^{T}\left(\int_{\mathcal{C}(-R)} u^{2}\right)^{(n+2) / 2}+\frac{C}{(R-\rho)^{4}} \int_{\mathcal{C}(-R)} \zeta_{-R}^{6}\left|\nabla u_{0}\right|^{2},
\end{aligned}
$$

we rewrite (4.6) as

$$
V(\rho)+(R-\rho)^{2} \cdot U(\rho) \leqslant \varepsilon(R-\rho)^{2} \cdot U(R)+F_{\varepsilon}(\rho, R)
$$

valid for $0 \leqslant \rho<R \leqslant R_{1}$.

An appeal to the iteration method presented in [16] entails the following estimate which holds for $\varepsilon>0$ sufficiently small with a new constant $K_{\varepsilon}$ dependent only on $\varepsilon$ :

$$
V(\rho)+\frac{(R-\rho)^{2}}{4} U(\rho) \leqslant K_{\varepsilon} F_{\varepsilon}(\rho, R) \quad \forall 0 \leqslant \rho<R \leqslant R_{1} .
$$

Rewriting in terms of $u$ and taking $\varepsilon>0$ sufficiently small, but fixed, we obtain

$$
\begin{aligned}
& \sup _{t \in(0, T)} \int_{\mathcal{C}(-\rho)} u^{2}(\cdot, t)+(R-\rho)^{2} \int_{0}^{T} \int_{\mathcal{C}(-\rho)}\left|\nabla u^{(n+2) / 6}\right|^{6} \\
& \quad \leqslant \frac{C}{(R-\rho)^{4+n N / 2}}\left[\int_{0}^{T}\left(\int_{\mathcal{C}(-R)} u^{2}\right)^{(n+2) / 2}+(R-\rho)^{n N / 2} \int_{\mathcal{C}(-R)} \zeta_{-R}^{6}\left|\nabla u_{0}\right|^{2}\right] .
\end{aligned}
$$

By virtue of the estimate

$$
\int_{0}^{T}\left(\int_{\mathcal{C}(-\rho)} u^{2}\right)^{(n+2) / 2} \leqslant T \cdot \sup _{t \in(0, T)}\left(\int_{\mathcal{C}(-\rho)} u^{2}\right)^{(n+2) / 2}
$$

we end up with

$$
\int_{0}^{T}\left(\int_{\mathcal{C}(-\rho)} u^{2}\right)^{\frac{n+2}{2}} \leqslant \frac{C \cdot T}{(R-\rho)^{\left(4+\frac{n N}{2}\right) \frac{n+2}{2}}}\left[\int_{0}^{T}\left(\int_{\mathcal{C}(-R)} u^{2}\right)^{\frac{n+2}{2}}+(R-\rho)^{\frac{n N}{2}} \int_{\mathcal{C}(-R)} \zeta_{-R}^{6}\left|\nabla u_{0}\right|^{2}\right]^{\frac{n+2}{2}} .
$$

Combining (4.3) with (4.1) and with (3.11), we estimate 


$$
\begin{aligned}
(R-\rho)^{n N / 2} \int_{\mathcal{C}(-R)} \zeta_{-R}^{6}\left|\nabla u_{0}\right|^{2} & \leqslant(R-\rho)^{n N / 2} \int_{B(0, R)} \zeta_{-R}^{6}\left|\nabla u_{0}\right|^{2} \\
& \leqslant K(\theta) \cdot R^{6+n N / 2} \int_{B(0, R)}\left|\nabla u_{0}\right|^{2} .
\end{aligned}
$$

This implies that

$$
\limsup _{R \rightarrow 0}(R-\rho)^{n N / 2} \int_{\mathcal{C}(-R)} \zeta_{-R}^{6}\left|\nabla u_{0}\right|^{2} \leqslant K(\theta) \cdot R^{4+N+2 \gamma+n N / 2} .
$$

With new coordinates $\xi=R_{1}-\rho$ and $\eta=R_{1}-R$ and with the function

$$
G(\xi):=\int_{0}^{T}\left(\int_{\mathcal{C}\left(\xi-R_{1}\right)} u^{2}\right)^{(n+2) / 2}
$$

(4.7) finally assumes the form

$$
G(\xi) \leqslant \frac{C \cdot T}{(\xi-\eta)^{(4+n N / 2)(n+2) / 2}}\left[G(\eta)+K(\theta) \cdot\left(R_{1}-\eta\right)^{4+N+2 \gamma+n N / 2}\right]^{(n+2) / 2}
$$

which holds for all $0 \leqslant \eta<\xi \leqslant R_{1}$. Applying the iteration Lemma A.2, we see that

$$
\int_{0}^{T}\left(\int_{\mathcal{C}(0)} u^{2}\right)^{(n+2) / 2}=G\left(R_{1}\right)=0
$$

provided (i) $T$ is such small that

$$
R_{1}^{\alpha} \geqslant C \cdot T \cdot\left(\int_{0}^{T}\left(\int_{\mathcal{C}\left(-R_{1}\right)} u^{2}\right)^{(n+2) / 2}+R_{1}^{4+N+2 \gamma+n N / 2}\right)^{n / 2} \quad \text { with } \alpha=\left(4+\frac{n N}{2}\right) \frac{n+2}{2}
$$

and (ii)

$$
4+N+2 \gamma+\frac{n N}{2} \geqslant \frac{(4+n N / 2)(1+n / 2)}{n / 2} .
$$

By the absolute continuity of Lebesgue's integral, (4.9) can be satisfied for $0<T<T^{*}$ with $T^{*}=$ $T^{*}\left(n, N, \gamma, u_{0}, \theta\right)$ sufficiently small.

Condition (4.10) is equivalent to the condition $\gamma \geqslant 4 / n$. This proves the theorem.

\section{A refined result on finite speed of propagation}

In this section, we use the Hardy-type inequality (3.5) to provide local qualitative results on finite speed of propagation for $n \in[2,3)$ in higher space dimensions also in the case that the support of initial data is not convex, but satisfies locally an exterior cone condition. In this way, previous results of [12] and [10] are essentially improved. For the ease of presentation, we confine ourselves again to the case of cones axial-symmetric to the $x_{N}$-axis. The general case follows easily by an rotation argument.

Theorem 5.1. Let $u$ be a solution of (1.1) to compactly supported non-negative initial data having $H^{1}$-regularity as constructed in Theorem 2.1. Assume that $y_{0}$ is a boundary point of $\operatorname{supp}\left(u_{0}\right)$. Let $\mathcal{C}\left(y_{0}, \theta\right):=\left\{x \in \mathbb{R}^{N}: \mid \bar{x}_{N}-\right.$ $\left.\bar{y}_{0 N} \mid<\left(x_{N}-y_{0 N}\right) \cdot \tan \theta\right\}, 0<\theta<\pi / 2$, be an infinite cone with opening angle $\theta$ such that $\operatorname{supp}\left(u_{0}\right) \cap \mathcal{C}\left(y_{0}, \theta\right)=\emptyset$. 
Consider for $R \geqslant 0$ cones

$$
\mathcal{C}\left(y_{0}+R \cdot e_{N}, \theta\right):=\left\{x \in \mathbb{R}^{N}:\left|\bar{x}_{N}-\bar{y}_{0 N}\right|<\left(x_{N}-\left(y_{0 N}+R\right)\right) \tan \theta\right\} .
$$

Then a continuous, increasing function $R:[0, \infty) \rightarrow \mathbb{R}_{0}^{+}, R(0)=0$, exists such that

$$
\operatorname{supp}(u(\cdot, t)) \cap \mathcal{C}\left(y_{0}+R(t) \cdot e_{N}, \theta\right)=\emptyset
$$

for all $t \in \mathbb{R}_{0}^{+}$.

In particular:

$$
R(T) \leqslant C(n, N, \theta) T^{1 / \alpha}\left(\int_{0}^{T}\left(\int_{C\left(y_{0}, \theta\right)} u^{2}\right)^{(n+2) / 2}\right)^{n /(2 \alpha)}
$$

with $\alpha=(8+n N)(n+2) / 4$.

Remark. (1) Note that - via Corollary 2.3 - the proof of Theorem 5.1 makes use of the weaker result on finite speed of propagation presented in [12] and [10].

(2) There exist still rather simple geometric settings which are not completely covered by the theorem, for instance $\operatorname{supp}\left(u_{0}\right)$ given by an annulus. To prove a corresponding result also in that case, a new version of a weighted energy estimate would be needed which replaces the terms in $u$ in estimate (2.19) by terms involving the product $\phi u$ where $\phi$ is an appropriate spatial localization function. In space dimension $N=1$, such an estimate is available (see [16]), in the multi-dimensional case its justification would be rather tedious. Therefore, we omit this here.

Proof. W.1.o.g. we may assume $y_{0}=0$. Choosing

$$
\zeta_{R}(x):= \begin{cases}\left(x_{N}-R\right)\left[\tan ^{2} \theta-\frac{\left|\bar{x}_{N}\right|^{2}}{\left(R-x_{N}\right)^{2}}\right] & x \in \mathcal{C}(R), \\ 0 & x \in \mathbb{R}^{N} \backslash \mathcal{C}(R)\end{cases}
$$

we infer by virtue of a similar reasoning as in the proof of Theorem 4.1 that

$$
\sup _{t \in(0, T)} \int_{\mathcal{C}(R)} \zeta_{R}^{4} u(\cdot, t)^{2}+\int_{0}^{T} \int_{\mathcal{C}(R)} \zeta_{R}^{6}\left|\nabla u^{(n+2) / 6}\right|^{6} \leqslant C \cdot \int_{0}^{T} \int_{\mathcal{C}(R)} u^{n+2}
$$

for all $R \geqslant 0$. As before, we estimate

$$
\zeta_{R}(x) \geqslant(R-\rho) \tan ^{2} \theta
$$

for all $x \in \mathcal{C}(\rho), \rho>R>0$, and we obtain by a similar iteration method as in the proof of Theorem 4.1

$$
\int_{0}^{T}\left(\int_{\mathcal{C}(\rho)} u^{2}\right)^{(n+2) / 2} \leqslant \frac{C \cdot T}{(\rho-R)^{(4+n N / 2)(n+2) / 2}}\left(\int_{0}^{T}\left(\int_{\mathcal{C}(R)} u^{2}\right)^{(n+2) / 2}\right)^{(n+2) / 2}
$$

for all $\rho>R>0$.

Writing

$$
G(\rho):=\int_{0}^{T}\left(\int_{\mathcal{C}(\rho)} u^{2}\right)^{(n+2) / 2}
$$


and using the classical iteration lemma of Stampacchia (see Lemma A.1), we find that $G(\rho)=0$ provided

$$
\rho^{\alpha} \geqslant C \cdot T\left(\int_{0}^{T}\left(\int_{\mathcal{C}(0)} u^{2}\right)^{(n+2) / 2}\right)^{n / 2}
$$

with $\alpha=(8+n N)(n+2) / 4$. Hence, (5.1) holds true.

\section{Acknowledgement}

This work has partially been supported by grant Gr1693/1-1 of the priority programme Benetzung und Strukturbildung an Grenzflächen of Deutsche Forschungsgemeinschaft.

\section{Appendix A}

In this section, we collect a couple of auxiliary results which were used in the paper. We begin with an iteration result by Stampacchia [21].

Lemma A.1 (Stampacchia's iteration lemma). Assume that a given non-negative, non-increasing function $G:\left(0, \rho_{0}\right) \rightarrow \mathbb{R}$ satisfies

$$
G(\xi) \leqslant \frac{c_{0}}{(\xi-\eta)^{\alpha}} G(\eta)^{\beta}
$$

for $0 \leqslant \eta<\xi \leqslant \rho_{0}$ and positive numbers $c_{0}, \alpha, \beta$ with $\beta>1$. Assume further that

$$
\rho_{0}^{\alpha} \geqslant 2^{\alpha \beta /(\beta-1)} \cdot c_{0} \cdot G(0)^{\beta-1} .
$$

Then, $G$ has a root in $\rho_{0}$.

The following modification was proposed by Dal Passo, Giacomelli and the author in [6].

Lemma A.2. Assume that a given non-negative, non-increasing function $G:\left(0, \rho_{0}\right) \rightarrow \mathbb{R}$ satisfies:

$$
G(\xi) \leqslant \frac{c_{0}}{(\xi-\eta)^{\alpha}}\left(G(\eta)+\left(\rho_{0}-\eta\right)^{\sigma}\right)^{\beta}
$$

for $0 \leqslant \eta<\xi \leqslant \rho_{0}$ and positive numbers $c_{0}, \alpha, \beta, \sigma$ such that

$$
\beta>1 \text { and } \sigma \geqslant \frac{\alpha}{\beta-1} \text {. }
$$

Assume further that

$$
\rho_{0}^{\alpha} \geqslant 2^{\alpha \beta /(\beta-1)}\left(1+2^{\alpha /(\beta-1)-\sigma}\right)^{\beta} \cdot c_{0} \cdot\left(G(0)+\rho_{0}^{\sigma}\right)^{\beta-1} .
$$

Then

$$
G\left(\rho_{0}\right)=0 .
$$

Finally, we need Gagliardo-Nirenberg's inequality in the following form (for a proof, we refer to [7]).

Lemma A.3. Let $1 \leqslant r \leqslant \infty, 0<q<p, m \in \mathbb{N}_{+}$such that

$$
\frac{1}{r}-\frac{m}{N}<\frac{1}{p}
$$


If $\Omega \subset \mathbb{R}^{N}$ is bounded with piecewise smooth boundary, then positive constants $c_{1}$ and $c_{2}$ depending only on $\Omega, r, p, m$ and $q$ exist such that for any $u \in L^{q}(\Omega)$ satisfying $D^{m} u \in L^{r}(\Omega)$, the following inequality holds:

$$
\|u\|_{p} \leqslant c_{1}\left\|D^{m} u\right\|_{r}^{a}\|u\|_{q}^{1-a}+c_{2}\|u\|_{q}
$$

where $a=\left(\frac{1}{q}-\frac{1}{p}\right) /\left(\frac{1}{q}+\frac{m}{N}-\frac{1}{r}\right)$.

Especially, if $\Omega$ is an infinite cone, i.e. for given points $x_{0}, y_{0} \in \mathbb{R}^{N}, x_{0} \notin B_{1}\left(y_{0}\right)$ a set

$C_{x_{0}, y_{0}}:=\left\{z \in \mathbb{R}^{N} \mid z=x_{0}+\lambda\left(y-x_{0}\right), y \in B_{1}\left(y_{0}\right), \lambda>0\right\}$,

then (A.4) holds with constants $c_{1}=c\left(\left\|x_{0}-y_{0}\right\|, r, p, m, q\right)$ and $c_{2}=0$.

\section{References}

[1] E. Beretta, M. Bertsch, R. Dal Passo, Nonnegative solutions of a fourth order nonlinear degenerate parabolic equation, Arch. Rational Mech. Anal. 129 (1995) 175-200.

[2] F. Bernis, Viscous flows, fourth order nonlinear degenerate parabolic equations and singular elliptic problems, in: J.I. Diaz, M.A. Herrero, A. Linan, J.L. Vazquez (Eds.), Free Boundary Problems: Theory and Applications, in: Pitman Research Notes in Mathematics, vol. 323, Longman, Harlow, 1995, pp. 40-56.

[3] F. Bernis, Finite speed of propagation and continuity of the interface for thin viscous flows, Adv. Differential Equations 1 (3) (1996) 337-368.

[4] M. Bertsch, R. Dal Passo, H. Garcke, G. Grün, The thin viscous flow equation in higher space dimensions, Adv. Differential Equations 3 (1998) 417-440.

[5] R. Dal Passo, H. Garcke, G. Grün, On a fourth order degenerate parabolic equation: global entropy estimates and qualitative behaviour of solutions, SIAM J. Math. Anal. 29 (1998) 321-342.

[6] R. Dal Passo, L. Giacomelli, G. Grün, A waiting time phenomenon for thin film equations, Ann. Scuola Norm. Sup. Pisa 30 (2001) 437-463.

[7] R. Dal Passo, L. Giacomelli, A. Shishkov, The thin film equation with nonlinear diffusion, Comm. Partial Differential Equations 26 (2001) $1509-1557$.

[8] P.G. de Gennes, Wetting: statistics and dynamics, Rev. Modern Phys. 57 (1985) 827-863.

[9] R. Ferreira, F. Bernis, Source-type solutions to thin-film equations in higher space dimensions, European J. Appl. Math. 8 (1997) 507-524.

[10] G. Grün, Droplet spreading under weak slippage: a basic result on finite speed of propagation, SIAM J. Math. Anal. 34 (2003) $992-1006$.

[11] G. Grün, On Bernis' interpolation inequalities in multiple space dimensions, Z. Anal. Anwendungen 20 (2001) 987-998.

[12] G. Grün, On free boundary problems arising in thin film flow, Habilitation thesis, University of Bonn, 2001.

[13] G. Grün, Droplet spreading under weak slippage: the optimal asymptotic propagation rate in the multi-dimensional case, Interfaces Free Bound. 4 (2002) 309-323.

[14] G.H. Hardy, Note on a theorem of Hilbert, Math. Z. 6 (1920) 314-317.

[15] G.H. Hardy, J.E. Littlewood, G. Pólya, Inequalities, Cambridge University Press, Cambridge, 1934.

[16] J. Hulshof, A. Shishkov, The thin film equation with $2 \leqslant n<3$ : finite speed of propagation in terms of the $L^{1}$-norm, Adv. Differential Equations 3 (1998) 625-642.

[17] W. Jäger, A. Mikelic, On the roughness-induced effective boundary conditions for an incompressible viscous flow, J. Differential Equations 170 (2001) 96-122.

[18] B. Opic, A. Kufner, Hardy-Type Inequalities, in: Pitman Research Notes, vol. 219, Longman, Harlow, 1990.

[19] A. Oron, S.H. Davis, S.G. Bankoff, Long-scale evolution of thin liquid films, Rev. Modern Phys. 69 (1997) $932-977$.

[20] F. Otto, Lubrication approximation with prescribed non-zero contact angle: an existence result, Comm. Partial Differential Equations 23 (1998) 2077-2164.

[21] G. Stampacchia, Équations elliptiques du second ordre à coefficients discontinus, Les presses de l'université de Montréal, Montréal, 1966. 